\title{
Clinical Characteristics and Neurological Findings of Pediatric Patients with Acute Carbon Monoxide Intoxication
}

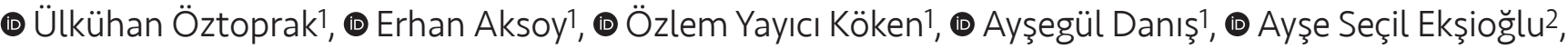 \\ (1) Nilden Tuygun 3 , (1) Deniz Yüksel1 \\ 1University of Health Sciences Turkey, Dr. Sami Ulus Training and Research Hospital, Clinic of Pediatric Neurology, Ankara, Turkey \\ 2University of Health Sciences Turkey, Dr. Sami Ulus Training and Research Hospital, Clinic of Pediatric Radiology, Ankara, Turkey \\ 3University of Health Sciences Turkey, Dr. Sami Ulus Training and Research Hospital, Clinic of Pediatric Emergency Medicine, Ankara, Turkey
}

\begin{abstract}
Aim: The aim of this study is to analyze children with acute carbon monoxide (CO) poisoning and to present two patients with rare neuroradiological findings.

Materials and Methods: We identified and reviewed the medical records of pediatric patients diagnosed with acute CO intoxication who were hospitalized in our department during a 10-year period. Epidemiologic and clinical data were collected and analyzed.

Results: A total of 326 children (166 girls, 160 boys; age range 1 to 17.8 years) with CO poisoning were identified. Their ages ranged from 1 to 17.8 years, with a mean of $8.3 \pm 4.8$ years. Improperly vented coal or wood stoves were the most common $(80.7 \%)$ cause of intoxication. The most common presenting symptoms were nausea/vomiting and headache. Seizure was seen in 32 patients (9.8\%). Two patients died and the mortality was $0.6 \%$. All patients received normobaric oxygen therapy until their carboxyhemoglobin (COHb) levels were decreased below $2 \%$ and their symptoms resolved. One hundred of the 326 patients (30.7\%) also were treated with hyperbaric oxygen (HBO) therapy as indicated by the signs and symptoms or $\mathrm{COHb}$ levels. Brain imaging was performed in 19 patients (thirteen magnetic resonance imaging and six computerized tomography), and was normal in 15. Acute brain stem demyelination related to water pipe smoking developed in one patient. All patients showed complete recovery without neurological sequelae except one who had mild right hemiparesis at discharge.

Conclusion: Acute CO intoxication is an important health problem in our country, especially in winter, because of poorly functioning heating systems. The clinical spectrum including neurological findings varies during childhood. We suggest that HBO therapy could be used safely in children. We believe that the combined administration of pulse methylprednisolone and $\mathrm{HBO}$ treatment might reduce cerebral damage caused by $\mathrm{CO}$ poisoning in selected pediatric patients.
\end{abstract}

Keywords: Acute carbon monoxide intoxication, clinical features, neurological findings, children

\section{Introduction}

Carbon monoxide (CO) causes direct cellular damage and tissue hypoxia by shifting the oxyhemoglobin dissociation curve to the left as it competes with oxygen for binding to hemoglobin. As a result, tissue oxygen extraction is hampered and oxidative stress causes tissue damage and clinical symptoms. $\mathrm{CO}$ is highly toxic for cardiac and cerebral tissue as these tissues have a higher metabolic

\section{Address for Correspondence}

Ülkühan Öztoprak MD, University of Health Sciences Turkey, Dr. Sami Ulus Training and Research Hospital, Clinic of Pediatric Neurology, Ankara, Turkey Phone: +90 5057807595 E-mail: ulkuhantoprak@yahoo.com ORCID: orcid.org/0000-0002-7309-3215

Received: 13.12 .2019 Accepted: 13.02 .2020

${ }^{(0)}$ Copyright 2021 by Ege University Faculty of Medicine, Department of Pediatrics and Ege Children's Foundation The Journal of Pediatric Research, published by Galenos Publishing House. 
rate and thus are sensitive to hypoxia (1). Symptoms of mild CO intoxication such as fatigue, headache, nausea and vomiting are non-specific. Visual disturbances, confusion, ataxia, seizure, loss of consciousness and death have also been reported in cases of moderate or severe exposure. Children are more sensitive to the toxic effects of $\mathrm{CO}$ and become symptomatic earlier than adults since their blood volume is lower, basal metabolic rate is higher and tissue oxygen demand is greater (2). There are few studies on CO poisoning in children, especially concerning neurological and neuroradiological findings. The purpose of this study is to describe and analyze the clinical features, treatment, neuroradiological findings and outcomes in children who were admitted with CO intoxication.

\section{Materials and Methods}

This study was performed retrospectively among pediatric patients who were admitted to the Emergency Department of Dr. Sami Ulus Training and Research Hospital due to CO poisoning between January 2007 and December 2017. Clinical and laboratory data were obtained from electronic hospital records retrospectively. Carboxyhemoglobin $(\mathrm{COHb})$ levels higher than $2 \%$ were considered as abnormal. A child having manifest symptoms of $\mathrm{CO}$ intoxication but a normal $\mathrm{COHb}$ level was accepted as acute $\mathrm{CO}$ poisoning if the child or family members had a history of $\mathrm{CO}$ exposure. Children with chronic pulmonary, cardiac, neurological or metabolic disease were excluded from this study. The age and gender of the patients, source of $\mathrm{CO}$ exposure, vital signs, Glasgow Coma scale (GCS) scores, laboratory results, treatment modalities, total hospital stay, results of computerized tomography (CT) and cranial magnetic resonance imaging (MRI) were recorded. Neurological abnormality was defined as altered consciousness, seizure, ataxia, or abnormal neurological examination findings at presentation. Cardiological abnormality was defined as changes in electrocardiography (ECG) and an increase in Troponin I or creatine kinase-myocardial band (CK-MB) levels and severe metabolic acidosis was defined as an arterial blood pH lower than 7.15.

All patients were treated with normobaric oxygen (NBO) therapy at a rate of $10 \mathrm{~L} / \mathrm{min}$ with a non-rebreathing face mask. The indications for hyperbaric oxygen (HBO) therapy were positive neurological symptoms (seizures, coma, lethargy, syncope) at presentation, refractory neurological symptoms after NBO therapy, $\mathrm{COHb}$ level greater than $25 \%$ or cardiac abnormality. A military based $\mathrm{HBO}$ therapy center in our city was consulted in cases of $\mathrm{HBO}$ requirement as our center lacks a HBO chamber.
This study was approved by the ethics committee of Ankara Training and Research Hospital Local Ethics Committee. Informed consent was obtained from the parents/care givers of the patients.

\section{Statistical Analysis}

Data analyses were performed using SPSS for Windows, version 22.0 (SPSS Inc., Chicago, IL, United States). Whether the distribution of continuous variables was normal or not was determined by Kolmogorov-Smirnov test. Levene test was used for the evaluation of homogeneity of variances. Unless specified otherwise, continuous data were described as mean \pm standard deviation for normal distributions, and median (range) for skewed distributions. Categorical data were described in terms of the number of cases (\%). Statistical analysis differences in normally distributed variables between two independent groups were compared by Student's t-test, Mann-Whitney $U$ test was applied for comparisons of non-normally distributed data. While the differences in normally distributed variables among more than two independent groups were analyzed by One-Way ANOVA, otherwise, Kruskal-Wallis test was applied for comparisons of the non-normally data.

\section{Results}

A total of 326 children (160 males and 166 females) with $\mathrm{CO}$ poisoning were included in this study. They were between 1 month and 17.8 years old, with a mean of $8.3 \pm 4.8$ years. The majority of poisonings happened during colder seasons (78.8\%). The distribution of the patients according to the source of intoxication is summarized in Table I.

One hundred and forty-four of the patients (44.1\%) arrived at the hospital on their own while 182 (55.8\%) patients were brought by an ambulance. Before admission, $49.4 \%$ of the patients had been given NBO therapy and $6.7 \%$ had received $\mathrm{HBO}$ therapy in another center. $\mathrm{COHb}$ levels varied between $0.1 \%$ and $47.5 \%$ with a mean value of $15.4 \pm 9.6 \%$. Fifty-one (15.6\%) patients had an initial CO level greater than $25 \%$. Forty-eight (14.7\%) patients were asymptomatic but were accepted as possible poisonings

Table I. The distribution of the patients according to source of intoxication $(n=326)$

\begin{tabular}{|l|l|}
\hline Source of exposure & Number of patients (\%) \\
\hline Coal or wood stoves & $263(80.7)$ \\
\hline Natural gas & $57(17.5)$ \\
\hline House fires & $5(1.5)$ \\
\hline Hookah & $1(0.3)$ \\
\hline
\end{tabular}


since other family members had overt CO poisoning. The distribution of initial symptoms and $\mathrm{COHb}$ levels are given in Table II.

While most patients had normal physical examination findings ( $n=310,95.1 \%), 9$ patients were lethargic, 6 patients were in coma and 1 patient had internuclear ophthalmoplegia. It was observed that asymptomatic cases had lower $\mathrm{COHb}$ levels with statistical significance than all findings except altered mental status $(p<0.05)$. Twentyone patients were admitted to the intensive care unit with a mean stay duration of $111.3 \pm 106.7$ hours (range: 10-480 hours). All patients with abnormal neurological examination and 5 patients with normal examination were followed up in the intensive care unit. One of these patients was a 1-month-old who had a plasma CO level of 19.4 and had seizures. Therefore, he was admitted to the intensive care unit for follow-up. The other 4 patients had a $\mathrm{COHb}$ level of over 25, and they had seizures prior to admission. Arterial blood gas measurements were performed in 281 patients, the mean pH level was 7.372 (range: 6.823-7.540); 3 children had severe metabolic acidosis. Lactate levels were elevated in 24 patients, CK-MB in 39 patients, troponin I in 30 patients and lactate dehydrogenase in 8 patients. No ECG abnormalities were detected except sinus tachycardia in two patients. Disseminated intravascular coagulation developed in five patients. Inhalation pneumonitis and acute respiratory distress syndrome developed in two of the five house fire victims. Two patients died because of sepsis and multiple organ failure. All surviving patients were discharged without sequelae except one patient with cerebrovascular ischemia.

All patients were treated with NBO therapy and 30.7\% $(n=100)$ with HBO therapy. The number of HBO treatment sessions varied between 1 and 16, with a mean of 2 and the mean $\mathrm{COHb}$ level was $17.2 \pm 13.1$ (range: $0.1-47.5$ ). The symptoms of patients who needed HBO therapy were syncope ( $n=42)$, a decreased level of consciousness ( $n=32)$, seizures $(n=26)$, headache $(n=19)$, nausea/vomiting $(n=19)$ and vertigo $(n=11)$. No HBO treatment related complications were observed.

Cranial imaging was performed in 19 patients (13 MRIs and 6 CTs) and 15 were normal. Abnormal findings in cranial scans were cerebellar tonsillar herniation due to severe brain edema in 2 patients, cerebrovascular ischemia in 1 patient, and brainstem demyelination with thalamic involvement in 1 patient. Detailed clinical information and cranial imaging findings are presented in Table III.

Two acute CO intoxication cases of interest along with their neurological and radiological findings are presented below.

\section{Case 1}

A 16-year old male was brought to the emergency department with headache and diplopia. Headache and nausea had begun after smoking two hookahs (water-pipes used to smoke tobacco) in a closed environment 24 hours earlier. He noticed that he had diplopia when he woke up the next morning. Neurological examination revealed impairment of adduction in the left eye and nystagmus in the right eye on the rightward gaze. His $\mathrm{COHb}$ level was $39 \%$ and NBO treatment was initiated. Cranial MRI scan performed at the $24^{\text {th }}$ hour revealed high intensity lesions at the left paramedian section of the pons, the medial segment of the left thalamus and left middle cerebellar peduncle on $\mathrm{T} 2$ sequences which showed mild contrast enhancement on $\mathrm{T} 1$ weighted sequences. The lesion at the left paramedian portion of the pons showed restricted diffusion on diffusion-weighted images. (Figure 1A-C). When cranial MRI and neurological examination findings were evaluated together, internuclear ophthalmoplegia due to $\mathrm{CO}$ intoxication was considered. $\mathrm{HBO}$ treatment with

Table II. Symptoms and signs of the patients on admission and COHb levels

\begin{tabular}{|l|l|l|}
\hline Symptoms and signs* & Number of patients (\%) & Mean COHb\% level (range) ${ }^{* *}$ \\
\hline Nausea/vomiting & $133(39.9)$ & $16.1(0.1-39.5)$ \\
\hline Headache & $121(37.1)$ & $16.3(0.7-39.9)$ \\
\hline Syncope & $69(21.2)$ & $17.9(0.1-47.5)$ \\
\hline Vertigo/dizziness & $47(14.4)$ & $17.4(0.3-30.8)$ \\
\hline Altered mental state & $42(12.9)$ & $12.7(0.1-47.5)$ \\
\hline Seizure & $32(9.8)$ & $19.1(0.3-45)$ \\
\hline Asymptomatic & $48(4.7)$ & $11.4(3-26)$ \\
\hline $\begin{array}{l}{ }^{*} \text { Many patients had more than one presenting symptom, }{ }^{* *} \text { Most of the patients received oxygen therapy (NBO or HBO) prior to admission to our center, COHb: } \\
\text { Carboxyhemoglobin, NBO: Normobaric oxygen, HBO: Hyperbaric oxygen }\end{array}$ \\
\hline
\end{tabular}




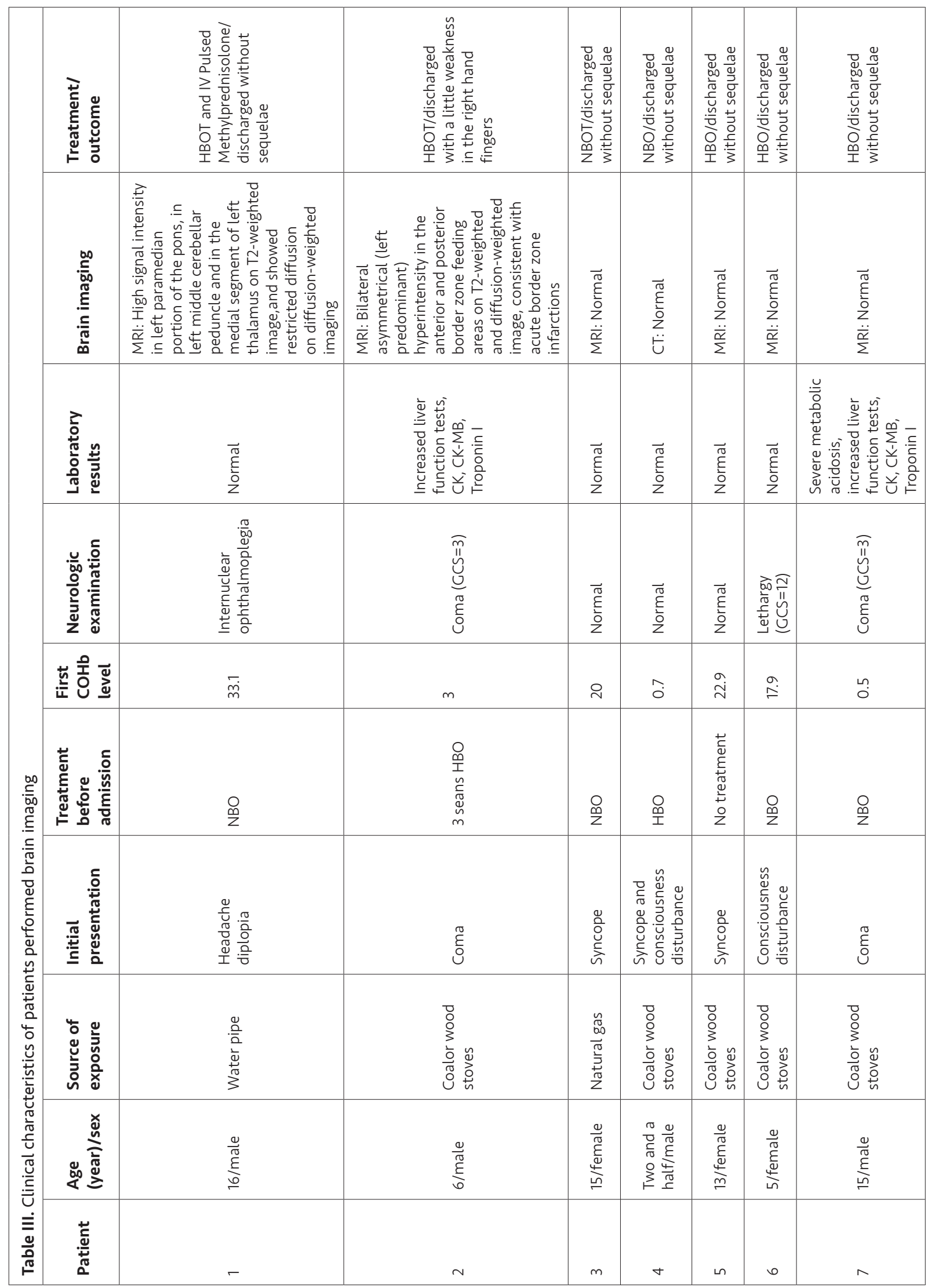




\begin{tabular}{|c|c|c|c|c|c|c|c|c|c|c|c|}
\hline 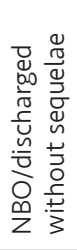 & $\stackrel{\bar{\omega}}{\overline{0}}$ & 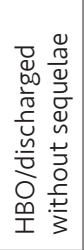 & 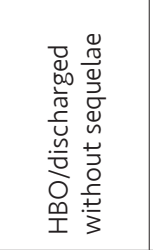 & 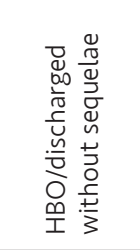 & 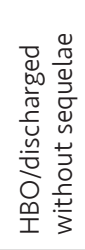 & 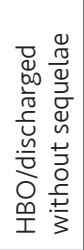 & $\stackrel{\overline{\ddot{\omega}}}{\overline{0}}$ & 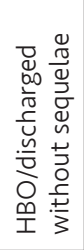 & 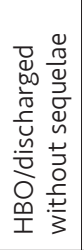 & 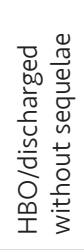 & 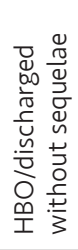 \\
\hline 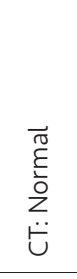 & 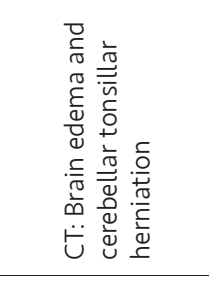 & $\begin{array}{l}\overrightarrow{\widetilde{\sigma}} \\
\stackrel{\xi}{\overline{0}} \\
\dot{z} \\
\dot{\ddot{\alpha}} \\
\stackrel{\Sigma}{\Sigma}\end{array}$ & $\begin{array}{l}\overline{\widetilde{\sigma}} \\
\sum_{\overline{0}} \\
\dot{\vec{\mu}} \\
\dot{\underline{\Sigma}}\end{array}$ & $\begin{array}{l}\overrightarrow{\widetilde{\sigma}} \\
\underline{\Sigma} \\
\dot{\Sigma} \\
\dot{\ddot{\Sigma}} \\
\dot{\Sigma}\end{array}$ & $\begin{array}{l}\overrightarrow{\widetilde{\sigma}} \\
\stackrel{\underline{y}}{2} \\
\dot{\ddot{\alpha}} \\
\dot{\Sigma}\end{array}$ & 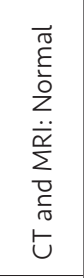 & 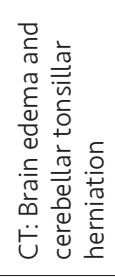 & $\begin{array}{l}\overrightarrow{\widetilde{\sigma}} \\
\stackrel{\xi}{0} \\
z \\
\dot{U} \\
\end{array}$ & $\begin{array}{l}\overline{\widetilde{\pi}} \\
\stackrel{\xi}{0} \\
\dot{z} \\
\dot{\ddot{\alpha}} \\
\dot{\Sigma}\end{array}$ & $\begin{array}{l}\overrightarrow{\widetilde{\sigma}} \\
\stackrel{\Xi}{0} \\
z_{\dot{U}} \\
\dot{U}\end{array}$ & $\begin{array}{l}\overrightarrow{\widetilde{\sigma}} \\
\underline{\xi} \\
\dot{0} \\
\dot{\ddot{\alpha}} \\
\frac{\dot{\alpha}}{\Sigma}\end{array}$ \\
\hline $\begin{array}{l}\overrightarrow{\widetilde{\sigma}} \\
\stackrel{\tilde{g}}{2}\end{array}$ & 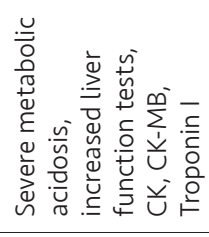 & $\begin{array}{l}\overrightarrow{\widetilde{\sigma}} \\
\stackrel{\xi}{\tilde{0}} \\
z\end{array}$ & 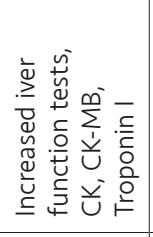 & 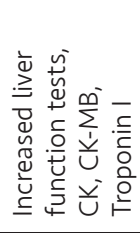 & 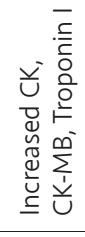 & 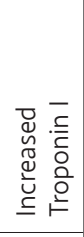 & 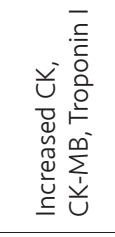 & 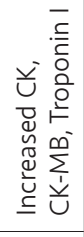 & 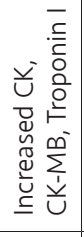 & $\begin{array}{l}\overrightarrow{\widetilde{\sigma}} \\
\stackrel{5}{0} \\
\dot{z}\end{array}$ & 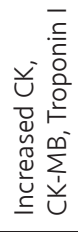 \\
\hline $\begin{array}{l}\stackrel{\widetilde{\sigma}}{\xi} \\
\frac{\partial}{2}\end{array}$ & 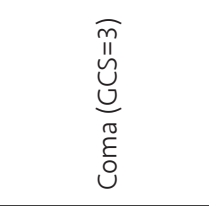 & $\begin{array}{l}\overrightarrow{\widetilde{\pi}} \\
\stackrel{\xi}{0} \\
\dot{z}\end{array}$ & 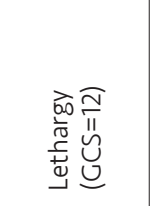 & $\begin{array}{l}\bar{\pi} \\
\ddot{u} \\
\underline{0} \\
\tilde{\tilde{\sigma}} \\
0\end{array}$ & $\begin{array}{l}\overrightarrow{\widetilde{\sigma}} \\
\stackrel{\xi}{0} \\
z\end{array}$ & $\begin{array}{l}\overrightarrow{\widetilde{\sigma}} \\
\stackrel{\xi}{0} \\
z\end{array}$ & $\begin{array}{l}\bar{\pi} \\
\dddot{u} \\
\underline{0} \\
\tilde{\tilde{v}} \\
0\end{array}$ & 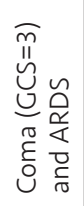 & 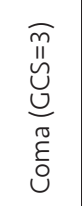 & $\begin{array}{l}\overrightarrow{\widetilde{\pi}} \\
\stackrel{\xi}{0} \\
z\end{array}$ & $\begin{array}{l}\overrightarrow{\widetilde{\sigma}} \\
\frac{\hat{y}}{z}\end{array}$ \\
\hline 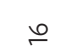 & $\stackrel{\circ}{\sim}$ & 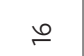 & $\stackrel{\stackrel{\infty}{\infty}}{\sim}$ & $\stackrel{\alpha}{m}$ & - & $\hat{m}$ & $\check{0}$ & m. & $\bar{\sim}$ & $\begin{array}{l}\stackrel{0}{\circ} \\
\stackrel{2}{n}\end{array}$ & $\check{\ddot{m}}$ \\
\hline 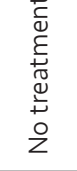 & 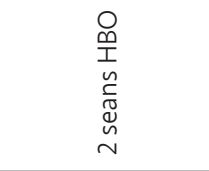 & $\begin{array}{l}\stackrel{0}{10} \\
\text { Z }\end{array}$ & $\begin{array}{l}0 \\
\text { 最 }\end{array}$ & $\begin{array}{l}\text { O } \\
\text { z }\end{array}$ & 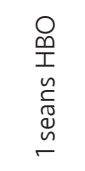 & 吕 & 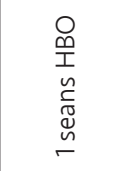 & 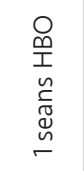 & 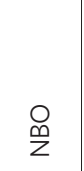 & 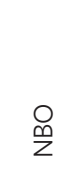 & $\begin{array}{l}\stackrel{O}{\mathrm{~m}} \\
\text { L }\end{array}$ \\
\hline 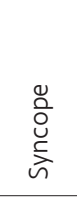 & $\stackrel{\tilde{\sigma}}{\tilde{U}}$ & $\begin{array}{l}\stackrel{0}{\bar{N}} \\
\stackrel{N}{N} \\
\end{array}$ & 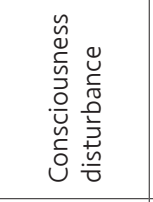 & 气 & 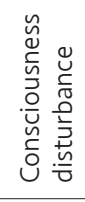 & 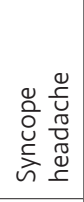 & $\stackrel{\tilde{\varepsilon}}{\tilde{U}}$ & $\stackrel{\widetilde{\sigma}}{\tilde{\Xi}}$ & $\stackrel{\tilde{\varepsilon}}{0}_{0}^{0}$ & $\begin{array}{l}\stackrel{\varrho}{\bar{N}} \\
\frac{N}{\sim}\end{array}$ & $\begin{array}{l}\stackrel{0}{\bar{N}} \\
\stackrel{\sim}{\tilde{N}} \\
\end{array}$ \\
\hline 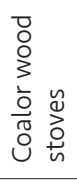 & 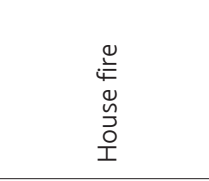 & 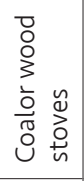 & 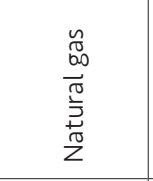 & 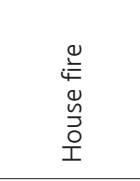 & 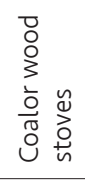 & 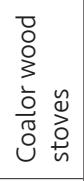 & 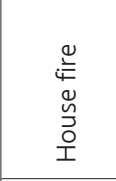 & 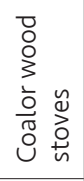 & 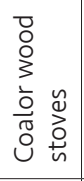 & 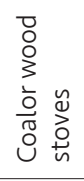 & 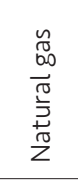 \\
\hline 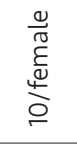 & 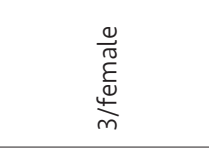 & 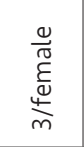 & 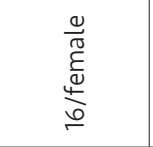 & 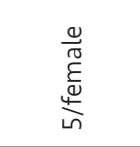 & 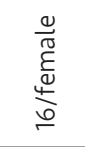 & 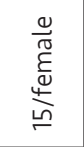 & 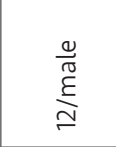 & 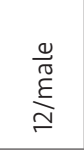 & 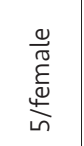 & 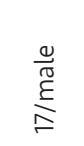 & $\begin{array}{l}\frac{0}{\sigma \sigma} \\
\frac{\xi}{\sigma} \\
\varrho\end{array}$ \\
\hline$\infty$ & $a$ & $\stackrel{\circ}{\circ}$ & $=$ & $\simeq$ & $m$ & $\doteq$ & $\stackrel{n}{\stackrel{1}{n}}$ & 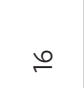 & $=$ & $\stackrel{\infty}{\leftarrow}$ & $\stackrel{\sigma}{\ulcorner}$ \\
\hline
\end{tabular}


simultaneous pulsed methylprednisolone $(30 \mathrm{mg} / \mathrm{kg} / \mathrm{day}$ for 5 days) was initiated. On the third day of steroid and $\mathrm{HBO}$ treatment, double vision resolved and the impairment of adduction in the left eye was significantly reduced. The patient was discharged without any sequelae after 7 days of hospitalization. MRI performed on the third week of followup revealed that the left cerebellar and left thalamic lesions had completely resolved and the size of the pontine lesion had significantly diminished. The patient's neurological examination at the third week was completely normal.

\section{Case 2}

A 6-year-old male patient had been found in an unconscious state in a room with a coal heater and was immediately taken to a local hospital. His GCS score was 5. He was immediately intubated and was given highflow oxygen therapy. His COHb level was $25.5 \%$. The patient was transferred to our center after one session of HBO treatment at another center in the same city. Upon arrival at our intensive care unit, his GCS score was 7 and he was being ventilated mechanically. His $\mathrm{COHb}$ level was $0.3 \%$ after 1 session of HBO treatment. Creatine phosphokinase was 41537 IU/L, CK-MB was 9605 $\mathrm{U} / \mathrm{L}$, aspartate aminotransferase was $605 \mathrm{U} / \mathrm{L}$, alanine aminotransferase was $260 \mathrm{U} / \mathrm{L}$, blood urea nitrogen was $33 \mathrm{mg} / \mathrm{dL}$, creatinine was $0.84 \mathrm{mg} / \mathrm{dL}$, lactate was 27.3 $\mathrm{mg} / \mathrm{dL}$, troponin I was $47 \mathrm{ng} / \mathrm{L}$, prothrombin time was 15.6 seconds, international normalized ratio was 2.29, D-dimer

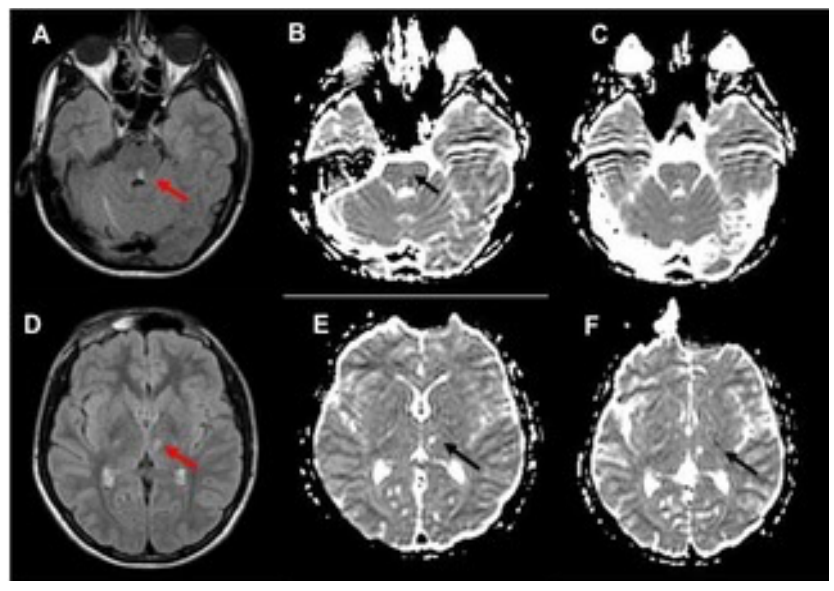

Figure 1. Initial $24 \mathrm{hr}$ cranial MR scan of a 16-year old male with CO poisoning secondary to water pipe smoking shows high signal intensity lesion in the left paramedian section of the pons on Flair (A) (red arrow) which also shows diffusion restriction on ADC (black arrow) and is resolved on $3^{\text {rd }}$ week control MR (C). Another lesion at the medial segment of the left thalamus on initial scan shows high signal on Flair (D) (red arrow) but no diffusion restriction on ADC initially (E) or on follow-up (F) (black arrows)

MR: Magnetic resonance, CO: Carbon monoxide, ADC: Apparent diffusion coefficient was $3373 \mathrm{ng} / \mathrm{mL}$. ECG and echocardiography were normal. After 72 hours, he gradually awakened and was extubated. On the $5^{\text {th }}$ day of follow-up, his muscle strength in upper extremity was $3 / 5$. Cranial CT revealed no abnormalities but MRI revealed bilateral asymmetrical (predominantly on the left) hyperintense lesions in the anterior and posterior border zones of the watershed areas of the middle cerebral arteries on T2-weighted and diffusion weighted sequences, consistent with acute watershed infarcts (Figure 2A-F). HBO treatment was administered in 16 sessions and physical therapy and rehabilitation were initiated. After 20 days, the patient was discharged from the hospital with physical therapy and rehabilitation recommendations. On the second year of follow-up, brain MRI was normal and neurologic examination revealed a slight weakness in the right-hand fingers. The patient was able to walk without support and did not have any difficulties at school.

\section{Discussion}

The incidence of CO poisoning depends on the region, geographical conditions, socio-economic status and seasons and it is more common in winter and in colder climates which are directly proportional to the need for heating. Stoves have been reported as the most common source of CO intoxication (3-5). Improperly vented coal or wood stoves were the most common (80.7\%) source of CO intoxication in our study.

Interestingly, one patient had double vision following hookah smoking and internuclear ophthalmoplegia was detected in neurologic examination. The hookah, also known as nargileh, shisha, water-pipe or hubble-bubble is used to smoke tobacco. In recent years, it has become increasingly popular among adolescents and young adults. A widespread misconception is that the water purifies the smoke, thereby rendering it harmless. However, this type of smoking produces the same harmful substances as cigarette smoking (tar, nicotine, $\mathrm{CO}$, etc.) and involves a serious risk of $\mathrm{CO}$ poisoning (6). A number of cases associated with

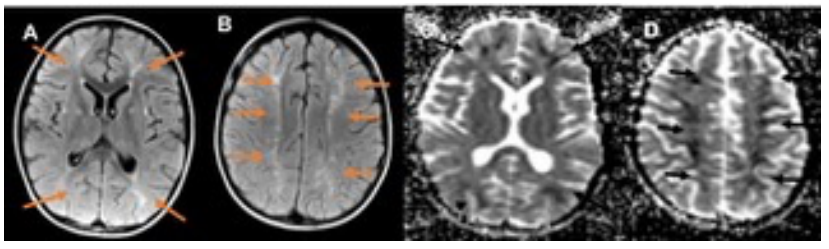

Figure 2. Cranial MRI of a 6-year old male intoxicated from stove shows bilateral asymmetrical hyperintensities in the anterior and posterior border zones on T2-weighted images (A-B) (red arrows) corresponding to areas of diffusion restriction on ADC images (C-D) (black arrows), consistent with acute border zone infarctions

MRI: Magnetic resonance imaging, ADC: Apparent diffusion coefficient 
hookah smoking have been previously reported (7-9). To the best of our knowledge, this is the first patient in the literature with internuclear ophthalmoplegia secondary to CO intoxication secondary to hookah smoking.

Children are more susceptible to the toxic effects of CO since they have fewer compensatory mechanisms against hypoxia and they need more oxygen due to their higher basal metabolic rate. Therefore, children become symptomatic earlier in the course of $\mathrm{CO}$ poisoning. Symptoms in pediatric patients are often non-specific, such as nausea and vomiting, and may be easily misdiagnosed as a viral infection $(10,11)$. In our study, we found that symptoms such as nausea, vomiting, headache, syncope and impaired consciousness were the most common symptoms. The prevalence of seizures in $\mathrm{CO}$ poisoning in children has been reported at rates ranging from $2.1 \%$ to $23.3 \%$ in the literature $(5,12,13)$. Seizures were observed in 32 cases $(9.8 \%)$ in our study. We observed that the mean $\mathrm{COHb}$ levels of the patients presented with syncope and seizure were relatively higher than the others. Lower $\mathrm{COHb}$ levels were detected compared to all findings except for altered mental status in asymptomatic patients. However, no definite clinical relationship was found between the $\mathrm{CO}$ levels and clinical symptoms of the patients. This finding had a correlation with previous studies. This is why our hospital is a tertiary center so that patients with neurological symptoms such as syncope, seizures and altered mental status will highly referred.

$\mathrm{COHb}$ levels were greater than $25 \%$ in 51 (15.6\%) patients and were normal in $23(7 \%)$ patients. Before admission to our center, $49.4 \%$ of the patients had been given NBO therapy and $6.7 \%$ had been given HBO therapy in another center. The half-life of $\mathrm{COHb}$ is $4-5$ hours in room air, and this decreases to 60 minutes when breathing $100 \%$ oxygen and further decreases to $15-30$ minutes during HBO therapy (14). We believe that those patients who applied to the emergency department with normal CO levels had longer transportation times while receiving 100\% oxygen treatment with a mask in the ambulance and thus, the measured values did not reflect the actual $\mathrm{COHb}$ levels. Therefore, we think that the presence of normal CO levels in children does not exclude the diagnosis of severe intoxication.

Tissue hypoxia is the main consequence of $\mathrm{CO}$ intoxication, so the basis of treatment is to give oxygen through the mask or in a hyperbaric chamber (15). HBO increases the dissolved oxygen level in the plasma thus enhancing oxygen delivery to the tissues. $\mathrm{HBO}$ also modulates mitochondrial oxidative metabolism, lipid peroxidation and neuronal apoptosis (16-19). Generally accepted indications for HBO treatment in children with acute $\mathrm{CO}$ poisoning are severe neurologic symptoms at presentation, continued neurologic symptoms after NBO therapy, myocardial ischemia and cardiac dysrhythmias, abnormal neuropsychiatric findings, high $\mathrm{COHb}$ levels and infants under six months with symptoms such as lethargy, irritability or poor feeding (20-22). It is recommended that the first session of $\mathrm{HBO}$ treatment be administered within 4-6 hours of poisoning and the recommended number of sessions is at least two $(23,24)$. In our study, all patients were treated with NBO and 100 patients (30.7\%) were treated with an average of 2 sessions of $\mathrm{HBO}$ treatment. $\mathrm{HBO}$ therapy was initiated within the first 24 hours and the most frequent indication for $\mathrm{HBO}$ treatment was syncope and altered mental status. HBO treatment is not free of side effects which include painful barotrauma, decompression sickness, pulmonary edema and hemorrhage, seizures and oxygen toxicity $(25,26)$. In our study, almost all patients who received $\mathrm{HBO}$ treatment were discharged in a healthy condition.

The nervous system is highly sensitive to the toxic effects of $\mathrm{CO}$. Some brain regions including the cerebral cortex, the white matter, the basal nuclei and the cerebellar Purkinje cells are highly sensitive to hypoxic damage. The globus pallidus is more prone to injury due to its high concentrations of heme-iron bound to $\mathrm{CO}$ and weak collateral blood supply $(27,28)$. Nineteen patients $(5.6 \%)$ underwent brain imaging and 13 patients had normal imaging. The typical globus pallidus involvement was not seen in any of the patients. One patient (Case 1) had acute brainstem demyelination, and 1 patient (Case 2) had acute watershed infarctions.

$\mathrm{CO}$ intoxication triggers inflammation and activation in $\mathrm{N}$-methyl-D-aspartate neurons, and the subsequent overactivity of neuronal nitric oxide synthase causes perivascular changes that cause neutrophil sequestration/ activation $(29,30)$. Xiang et al. (31) stated that inflammation plays an important role in delayed encephalopathy induced by acute $\mathrm{CO}$ poisoning in rats and can be attenuated by dexamethasone by protecting myelin from inflammatory damage. In a recent study with adult patients, it was found that the combined application of dexamethasone and $\mathrm{HBO}$ therapy could yield better efficacy for patients than HBO therapy as a monotherapy (32). Pulse methylprednisolone therapy can be given with various indications such as acute demyelinating disorders, cerebral vasculitis and encephalopathy. High doses of steroid is believed to suppress inflammation, edema and demyelination. The 
patient with internuclear ophthalmoplegia and acute brainstem demyelination was given pulse steroid therapy combined with $\mathrm{HBO}$ treatment. On the third day of this treatment regime, we observed that the patient's double vision disappeared and the impairment of adduction in the left eye had significantly subsided. We believe that the combined administration of corticosteroid and $\mathrm{HBO}$ treatment might reduce cerebral damage caused by $\mathrm{CO}$ poisoning.

\section{Study Limitations}

The study center is a research and education center, and some of the patients were referred from other hospitals. The patients referred from other hospitals were treated with oxygen during the transfer. Therefore, these patients' measured $\mathrm{COHb}$ values may be lower than their actual $\mathrm{COHb}$ values. Also, the absence of an institutional protocol of HBO therapy is another limitation of our study.

\section{Conclusion}

In this study, we presented our clinical experience in childhood $\mathrm{CO}$ poisonings with a large number of cases. In conclusion, acute neurologic manifestations following $\mathrm{CO}$ exposure are common in children. Detailed history, physical examination and a high level of suspicion are important in the diagnosis of $\mathrm{CO}$ poisoning. HBO therapy can be safely used in children who have $\mathrm{CO}$ poisoning. A combined treatment regime consisting of pulse methylprednisolone and HBO treatment may have a more therapeutic potential to prevent neuronal damage in selected pediatric patients with CO poisoning.

\section{Ethics}

Ethics Committee Approval: This study was approved by the ethics committee of Ankara Training and Research Hospital Local Ethics Committee.

Informed Consent: Informed consent was obtained from the parents/care givers of the patients.

Peer-review: Externally peer-reviewed.

\section{Authorship Contributions}

Concept: Ü.Ö., Design: Ü.Ö., D.Y., N.T., Data Collection or Processing: Ü.Ö., Ö.Y.K, E.A., Analysis or Interpretation: Ü.Ö., Ö.Y.K, E.A., D.Y., Radiological Evaluation: A.S.E., Literature Search: Ü.Ö., A.D., E.A., Writing: Ü.Ö., Ö.Y.K.

Conflict of Interest: No conflict of interest was declared by the authors.

Financial Disclosure: The authors declared that this study received no financial support.

\section{References}

1. Ernst A, Zibrak JD. Carbon monoxide poisoning. N Engl / Med 1998; 339:603-8.

2. Kind T. Carbon monoxide. Pediatr Rev 2005; 26:150-1.

3. Mendoza JA, Hampson NB. Epidemiology of severe carbon monoxide poisoning in children. Undersea Hyperb Med 2006; 33:439-46.

4. Yurtseven S, Arslan A, Eryigit U, et al. Analysis of patients presenting to the emergency department with carbon monoxide intoxication. Turk J Emerg Med 2016; 15:159-62.

5. Damlapinar R, Arikan Fl, Sahin S, Dallar Y. Lactate level is more significant than carboxihemoglobin level in determining prognosis of carbon monoxide intoxication of childhood. Pediatr Emerg Care 2016; 32:377-83.

6. Blank MD, Cobb CO, Kilgalen B, et al. Acute effects of waterpipe tobacco smoking: a double-blind, placebo-control study. Drug Alcohol Depend 2011; 116:102-9.

7. Veen M. Carbon monoxide poisoning caused by water pipe smoking: a case series. J Emerg Med 2016; 51:41-4.

8. Von Rappard J, Schönenberger M, Bärlocher L. Carbon monoxide poisoning following use of a water pipe/hookah. Dtsch Arztebl Int 2014; 111:674-9.

9. Türkmen S, Eryigit U, Sahin A, Yeniocak S, Turedi S. Carbon monoxide poisoning associated with water pipe smoking. Clin Toxicol (Phila) 2011; 49:697-8.

10. Crocker PJ, Walker JS. Pediatric carbon monoxide toxicity. I Emerg Med 1985; 3:443-8.

11. Widdop B. Analysis of carbon monoxide. Ann Clin Biochem 2002; 39:378-91.

12. Sethuraman KN, Douglas TM, Bostick BB, Comer AC, Myers B, Rosenthal RE. Clinical Characteristics of Pediatric Patients With Carbon Monoxide Poisoning. Pediatr Emerg Care 2020; 36:178-81.

13. Cho $\mathrm{CH}$, Chiu NC, Ho CS, Peng CC. Carbon monoxide poisoning in children. Pediatr Neonatol 2008; 49:121-5.

14. Kao LW, Nañagas KA. Carbon monoxide poisoning. Emerg Med Clin North Am 2004; 22:985-1018.

15. Prockop LD, Chichkova RI. Carbon monoxide intoxication: an updated review. I Neurol Sci 2007; 262:122-30.

16. Annane D, Chadda K, Gajdos P, Jars-Guincestre MC, Chevret S, Raphael JC. Hyperbaric oxygen therapy for acute domestic carbon monoxide poisoning: two randomized controlled trials. Intensive Care Med 2011; 37:486-92.

17. Xue L, Wang WL, Li Y, et al. Effects of hyperbaric oxygen on hippocampal neuronal apoptosis in rats with acute carbon monoxide poisoning. Undersea Hyperb Med 2017; 44:121-31.

18. Thom SR, Bhopale VM, Fisher D. Hyperbaric oxygen reduces delayed immune-mediated neuropathology in experimental carbon monoxide toxicity. Toxicol Appl Pharmacol 2006; 213:152-9.

19. Thom SR. Functional inhibition of leukocyte B2 integrins by hyperbaric oxygen in carbon monoxide-mediated brain injury in rats. Toxicol Appl Pharmacol 1993; 123:248-56.

20. Tibbles PM, Edelsberg IS. Hyperbaric-oxygen therapy. N Engl I Med 1996; 334:1642-8. 
21. Tomaszewski CA, Thom SR. Use of hyperbaric oxygen in toxicology. Emerg Med Clin North Am 1994; 12:437-59.

22. Liebelt EL. Hyperbaric oxygen therapy in childhood carbon monoxide poisoning. Curr Opin Pediatr 1999; 11:259-64.

23. Gorman DF, Clayton D, Gilligan JE, Webb RK. A longitudinal study of 100 consecutive admissions for carbon monoxide poisoning to the Royal Adelaide Hospital. Anaesth Intensive Care 1992; 20:311-6.

24. Weaver LK, Hopkins RO, Chan KJ, et al. Hyperbaric oxygen for acute carbon monoxide poisoning. N Engl J Med 2002; 347:105767.

25. Norkool DM, Kirkpatrick JN. Treatment of acute carbon monoxide poisoning with hyperbaric oxygen: a review of 115 cases. Ann Emerg Med 1985; 14:1168-71.

26. Kao LW, Nañagas KA. Toxicity associated with carbon monoxide. Clin Lab Med 2006; 26:99-125.

27. Beppu T. The role of MR imaging in assessment of brain damage from carbon monoxide poisoning: a review of the literature. AJNR Am / Neuroradiol 2014; 35:625-31.
28. Durak AC, Coşkun A, Yıkılmaz A, Erdoğan F, Mavili E, Guven $M$. Magnetic resonance imaging findings in chronic carbon monoxide intoxication. Acta Radiol 2005; 46:322-7.

29. Thom SR. Carbon monoxide-mediated brain lipid peroxidation in the rat. ) Appl Physiol 1990; 68:997-1003.

30. Thom SR, Fisher D, Zhang I, Bhopale VM, Cameron B, Buerk DG. Neuronal nitric oxide synthase and $\mathrm{N}$-methyl-D-aspartate neurons in experimental carbon monoxide poisoning. Toxicol Appl Pharmacol 2004; 194:280-95.

31. Xiang WP, Xue H, Wang BJ. Delayed encephalopathy of acute carbon monoxide intoxication in rats: potential mechanism and intervention of dexamethasone. Pak / Pharm Sci 2014; 27:2025-8

32. Xiang $W$, Xue $H$, Wang $B$, et al. Combined application of dexamethasone and hyperbaric oxygen therapy yields better efficacy for patients with delayed encephalopathy after acute carbon monoxide poisoning. Drug Des Devel Ther 2017; 11:513-9. 